PAPER

\section{Water wave measurement from stereo images of specular reflections}

To cite this article: Daniel Kiefhaber et al 2015 Meas. Sci. Technol. 26115401

View the article online for updates and enhancements.
You may also like

- Simulation of coastal wave spectra energy
from ENVISAT satellite data
Maged Marghany
- Fast Simulation Method for Ocean Wave
$\frac{\text { Base on Ocean Wave Spectrum and }}{\text { Improved Gerstner Model with GPU }}$
$\frac{\text { Wenqiao Zhang, Jing Zhang and Tianchi }}{\text { Zhang }}$
- Characterisation of a multi-view fringe
projection system based on the stereo
matching of rectified phase maps
A Shaheen, D Sims-Waterhouse, P
Bointon et al.

\section{Recent citations}

- Application of photogrammetry for spatial
$\frac{\text { free surface elevation and velocity }}{\text { measurement in wave flumes }}$
Alan Fleming et al
- Influence of natural surfactants on short
$\frac{\text { wind waves in the coastal Peruvian waters }}{\text { D. Kiefhaber et al }}$




\title{
Water wave measurement from stereo images of specular reflections
}

\author{
Daniel Kiefhaber ${ }^{1,2}$, Guillemette Caulliez ${ }^{3}$, Christopher J Zappa ${ }^{4}$, \\ Julia Schaper ${ }^{1,2}$ and Bernd Jähne ${ }^{1,2}$ \\ ${ }^{1}$ Institute of Environmental Physics, University of Heidelberg, Im Neuenheimer Feld 229, 69115 \\ Heidelberg, Germany \\ ${ }^{2}$ Heidelberg Collaboratory for Image Processing, University of Heidelberg, Speyerer Str. 6, 69117 \\ Heidelberg, Germany \\ ${ }^{3}$ Mediterranean Institute of Oceanography, 13288 Marseille, France \\ ${ }^{4}$ Lamont-Doherty Earth Observatory, Columbia University, 61 Route 9W, Palisades, NY 10964, USA \\ E-mail: daniel.kiefhaber@iup.uni-heidelberg.de
}

Received 31 March 2015, revised 9 July 2015

Accepted for publication 28 July 2015

Published 1 October 2015

\begin{abstract}
A new optical instrument for the study of ocean waves, the Reflective Stereo Slope Gauge, has been developed. Its purpose is to measure ocean wave field parameters that are crucial to the air-sea exchange of momentum, heat and gases. The instrument combines a statistical wave slope measurement method similar to Cox and Munk's sun glitter technique with a dedicated stereo camera and associated illumination setup for direct wave height measurements. The instrument output was validated under controlled conditions in a wind-wave facility.
\end{abstract}

Keywords: wave slope statistics, stereo photography, specular reflections

(Some figures may appear in colour only in the online journal)

\section{Introduction}

Short ocean waves with wavelengths ranging from centimetres to metres are important for a number of reasons. The roughness of the sea surface at these scales is strongly correlated with the rate of the exchange of momentum, heat and gases between the atmosphere and the ocean (Jähne et al 1987, Zappa et al 2004). Furthermore, the typical scale of this roughness is comparable to the wavelength of electromagnetic waves used in microwave remote sensing techniques. Therefore, short wind waves have a fundamental influence on the backscattered radar power intensity (Valenzuela 1978).

In contrast to long wavelength swell, which is created mainly through non-linear wave-wave interactions, short waves are mostly generated by the local wind stress. However, the local momentum input into the wave field also depends on longer waves and the 'history' of the interaction of the wind and waves (Edson et al 2013). To investigate the complex interactions between wind, waves and the exchange of momentum, heat and gases between the atmosphere and the ocean, it is crucial to have instrumentation to reliably measure representative wave field quantities on all wave scales.

For studies on air-sea interaction, a time-resolved characterisation of the sea surface topography is often neither required nor attainable and the measurement of a few statistical parameters is sufficient. Larger scale waves are commonly described by the significant wave height (which is proportional to the standard deviation of the water surface elevation), as well as the frequency of the dominant swell and wind waves. A key parameter in the description of the short wave field, particularly for applications in air-sea interaction, is the mean square slope of the wavy surface, $\sigma_{\mathrm{s}}^{2}$ (Frew et al 2004).

The Reflective Stereo Slope Gauge (RSSG), the instrument presented here, is able to measure the most important wave parameters under a wide range of environmental conditions. It was designed to be used in the field, on research platforms or vessels. In this paper, after a short overview on the available instrumentation (section 1.1), the physical principles of the RSSG height and slope measurement are described (sections 2.1 and 2.2) and the general configuration of the instrument is presented (section 2.3). The results of a feasibility 
experiment in a laboratory wind wave facility are presented and the capabilities and limitations of the instrument are discussed (section 3).

\subsection{Short review of wave imaging techniques}

1.1.1. Stereo photography. Optical wave measurements date back to the beginning of the 20th century, when German photographers were using elaborate stereo camera setups to chart colonial coastlines. Incidentally, they found that information about wave amplitude and wavelength could be extracted from stereo photographs (Laas 1905, 1906, Kohlschütter 1906). A systematic characterisation of water surface elevation by stereo photography was attempted by Schumacher (1939), who had already recorded time series at a frequency of $1 \mathrm{~Hz}$ (Schumacher 1950). Shortly after Schumacher reported on the German experiments at a symposium in Washington DC. Schumacher (1952), a massive American collaboration, the Stereo Wave Observation Project (SWOP) was launched. Stereo images were taken from two planes flying in formation, while reference measurements were conducted aboard a research vessel. The manual evaluation of the images turned out to be costly and time consuming and despite tremendous efforts, both financially and in terms of manpower, only 2 of 100 image pairs were evaluated. However, they yielded the first record of directional wave spectra (Cote et al 1960, Kinsman 1965).

Later attempts to extend the measurement of wave spectra to short gravity waves and beyond, e.g. by Dobson (1970), Holthuijsen (1983a, 1983b), Shemdin et al (1988) and Banner et al (1989) all suffered from the complexity of the stereo evaluation process, which resulted in limited numbers of processed images. With the progress in computing power, image processing is not a severe restriction on the applicability of stereo photography to wave imaging anymore, as demonstrated in more recent studies (e.g. Benetazzo 2006, Kosnik and Dulov 2011).

1.1.2. Optical wave slope measurements. In addition to stereo photography, a number of specialised optical techniques have been developed for studying water waves. Most of them do not determine the water surface elevation, but measure wave slope, i.e. the spatial derivative of the surface elevation. Wave slope has proven to be in the same order of magnitude for all wave scales, whereas wave amplitude ranges from less than one millimetre to a few metres. Elaborate techniques have been developed based on the refraction of light at the water surface (e.g. Cox 1958, Hughes et al 1977, Jähne and Reimer 1990, Bock and Hara 1992, Hwang et al 1993, Zhang et al 1996, Kiefhaber et al 2014). By concept, all these techniques require optical components (e.g. cameras or light sources) to be submerged into the water, which complicates employing them in field studies.

Reflection-based methods have the advantage of being non-intrusive and a number of experiments were conducted using sun glitter (Cox and Munk 1954a), diffuse sky light (Stilwell 1969) or artificial light sources (Schooley 1954,
Wu 1971, Shaw and Churnside 1997) for lighting. More recently, Zappa et al (2008) presented an instrument that measures the two components of wave slope by taking advantage of the Fresnel reflection coefficient's dependence on polarisation. Open ocean results using this polarimetric camera showed high wavenumber spectral characteristics of the ocean surface roughness above $2000 \mathrm{rad} \mathrm{m}^{-1}$ (Zappa et al 2012).

For more information on the capabilities and shortcomings of different concepts for optical wave slope measurement, the reader is referred to Zhang et al (1996) and Jähne et al (1994).

1.1.3. The need for a new instrument. For systematic studies of air-sea exchange processes in the field, it is necessary to measure the wave field under varying conditions, day and night. This excludes all passive techniques based on the reflection of skylight or sun glitter. Furthermore, all refraction-based instruments need to be partly submerged, which complicates experimental design and likely disturbs the nearsurface dynamics.

To overcome the fundamental shortcomings of the existing instruments and techniques, a new instrument, named the RSSG, was developed. It is loosely based on the Cox and Munk (1954a) (statistical) sun glitter slope measurement technique but uses artificial light sources to allow day- and night-time measurements. The RSSG also features wave height measurements by stereo triangulation. Thus, it allows one to obtain information on both long waves (wave height time series) and short waves (wave slope statistics).

\section{Method}

In this chapter, the measurement principles for wave height (section 2.1) and wave slope statistics (section 2.2) are detailed and a description of the measuring device is given (section 2.3) before detailing the key steps of the data processing routine in section 2.4 .

\subsection{Height measurement}

Stereo triangulation requires knowledge of the stereo disparity, i.e. the shift between the two image positions of an object (Hartley and Zisserman 2003). One way of determining the disparity consists of selecting a small area of interest in one image and finding the closest representation of this area in the other image (block matching). This requires that objects look very similar in both images.

The water surface is a specular reflector, which means that all reflections are directed, the angle of the reflected ray with respect to the local surface normal is equal to the incidence angle. The image of the water surface thus not only depends on the shape of the surface itself, but also on the characteristics of the light source (which may be natural, e.g. the sky, the sun, or artificial), which complicates finding corresponding points, unless a special setup is used, which will be detailed below. 
a

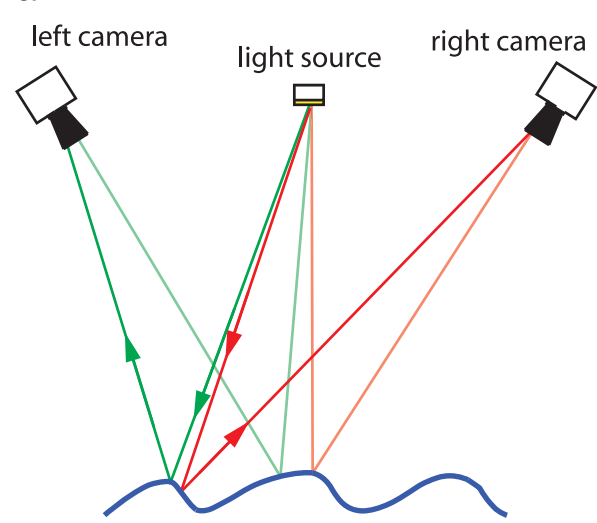

b

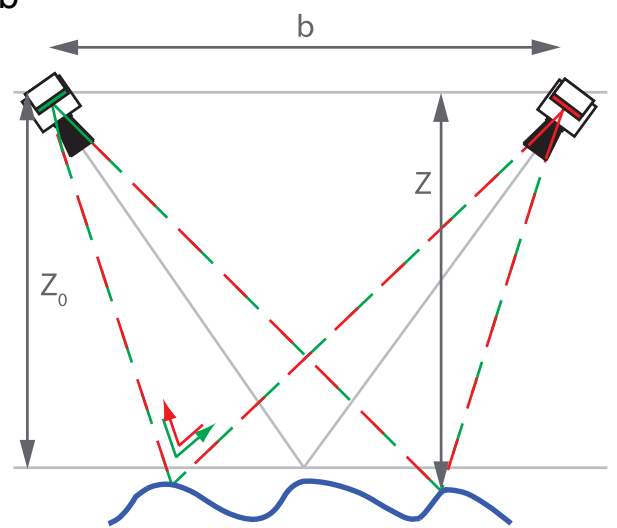

Figure 1. (a) With a randomly positioned (e.g. natural) light source, reflections observed by the two stereo cameras come from different surface positions, leading to a bias in the determined stereo disparity. (b) Using two light sources, the problem can be solved by inverting the path of the light rays, the right camera sees reflections from the left light source, and vice versa.

2.1.1. Stereo imaging of specular surfaces. Waves are visible in photographs of the water surface due to a combination of two effects; (1) the reflection coefficient depends on the incidence angle and (2) the brightness of the sky is often inhomogeneous. What a camera sees is not the surface itself, but a distorted reflection of the incident (sky)light, because the water surface is a fully specular reflector.

The consequence of inhomogeneous illumination was already pointed out by Jähne et al (1994) and is demonstrated in figure 1(a): The light from a randomly positioned point light source is reflected into both the left and the right camera. Due to the different viewing angles of the cameras, the points on the water surface at which the light is reflected into the two cameras are not identical. As a consequence, the zones on the water surface in which light is reflected into the left and right camera have different shapes and positions. In a block matching approach to estimating the stereo disparity, this phenomenon causes unwanted errors.

If additional constraints are introduced (such as the smoothness and continuity of the water surface), stereo disparities can be estimated in spite of the aforementioned problems. Several groups have succeeded in developing algorithms to deal with the consequences of the specularity of the water surface, e.g. Gallego et al (2011), Benetazzo et al (2012), Mironov et al (2012), Fedele et al (2013).

We choose a different approach for stereo imaging at the water surface: by utilising Helmholtz stereopsis (Helmholtz 1867), which was also used by Zickler et al (2002) for facial stereo vision, it becomes possible to get images in which finding corresponding points is greatly simplified and intricate algorithms are not required. This approach is shown in figure 1(b): by using two light sources, the path of the light rays entering the two cameras can be the same, with light propagating in opposing directions. Reflections of the right light source seen by the left camera, and vice versa, thus come from the same locations on the water surface. This way, the bias that was present with one (point) light source is avoided and the reflections have an almost identical shape in both images. A first instrument based on this principle was developed by Waas and Jähne (1992).
2.1.2. Water elevation from stereo disparity. Solving the correspondence problem of stereo triangulation, i.e. identifying the position of the same object in both images to compute the disparity, is straightforward for speckle images acquired with a setup similar to that in figure 1(b). The water surface is continuous and exhibits no occlusions (except for wave breaking events), the reflections are small and bright with a dark background, thus their position can be determined with high precision. A typical example is given in figure 2(a), where a false colour overlay is used to show both the left and right camera images. The saturated magenta and cyan colours correspond to the high intensity of the reflected light, while the originally dark background is shown in white. We use a block matching approach and determine the disparity $d$, see figure 2(b), by finding the maximum of the cross correlation between small interrogation windows containing multiple reflections (see section 2.4.2).

Stereo data processing commonly makes use of epipolar geometry after a full calibration of the stereo cameras, including their translation and rotation as well as intrinsic parameters such as the lenses' focal length (Hartley and Zisserman 2003). In our system, a simplified approach was chosen to facilitate calibration of the instrument, particularly during field experiments. The dependence of the disparity of objects in the stereo images on object distance for a setup with tilted optical axes (rotation of the cameras around the axis orthogonal to the plane spanned by the stereo base and the camera's optical axis) is deduced in Kiefhaber (2010). For a given disparity $d$ at the image coordinate $u$ (image axis parallel to the stereo base line $b$ ), the distance $Z$ to the water surface is ${ }^{1}$

$$
Z=-\frac{\mathrm{b}\left(4 f^{2} \mathrm{Z}_{0}^{2}-2 d \mathrm{~b} f \mathrm{Z}_{0}+d u \mathrm{~b}^{2}-u^{2} \mathrm{~b}^{2}\right)}{-4 d f \mathrm{Z}_{0}^{2}-\left(4 \mathrm{~b} u^{2}-4 d \mathrm{~b} u+4 \mathrm{~b} f^{2}\right) \mathrm{Z}_{0}+d f \mathrm{~b}^{2}},
$$

with $f$, the focal length of the lenses and $\mathrm{Z}_{0}$, the stereo reference distance (distance at which the two optical axes intersect, see figure 1(b)). As will be shown in section 3, the accuracy of

${ }^{1}$ Here and in the following, Roman font is used for fixed parameters, while variables are set in italic. 
a

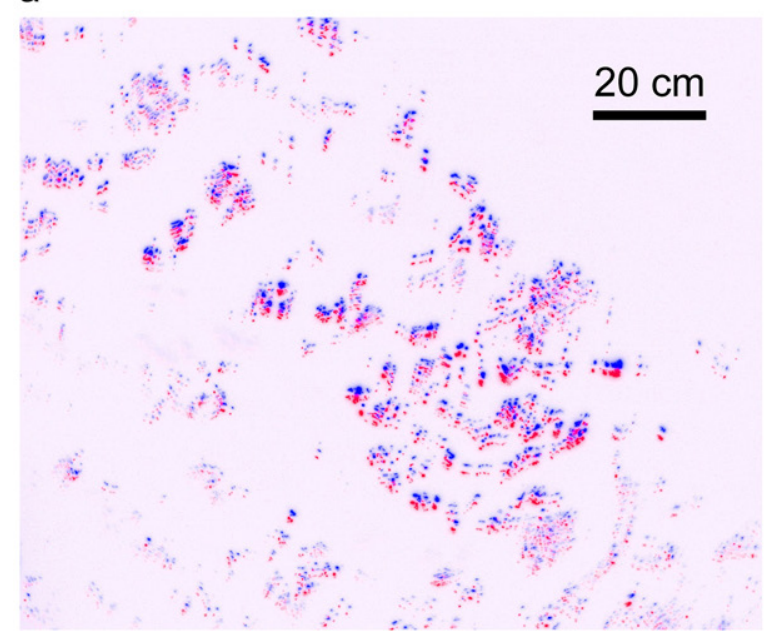

b

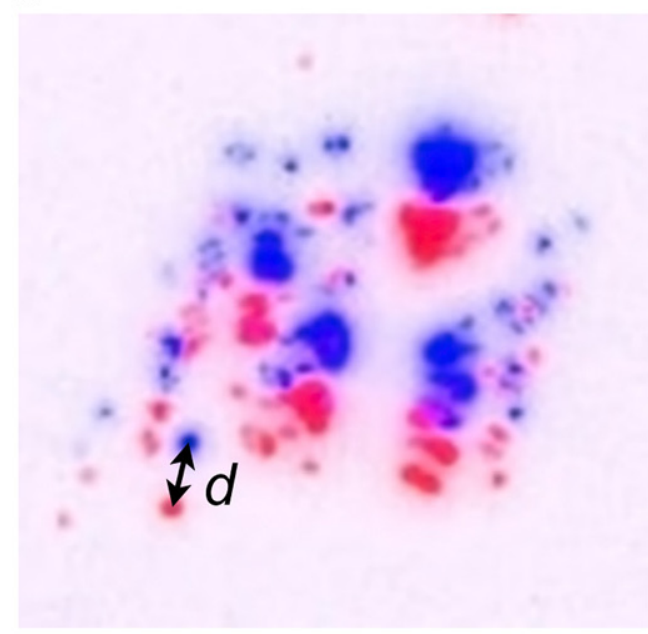

Figure 2. (a) Example of specular reflections of near point light sources at the ocean surface acquired with the RSSG. False colour overlay of the left and right camera images. (b) Detail from the stereo image pair (magnified by a factor of 10). The shift between the positions of an object in the two images is the stereo disparity $d$.

the camera alignment in our instrument is sufficient for such a simplified model to give correct results without extensive calibration.

\subsection{Slope statistics measurement}

The slope measurement technique used in this study is related to the classic sun glitter technique (Cox and Munk 1954b) and the work of Schooley (1954), who analysed reflections from a flash bulb. We also use an artificial light source to allow measurements under a wider range of environmental conditions, day and night.

2.2.1. Geometry of reflection. The principle of measurement is illustrated in figure 3: a downward-looking camera is observing reflections of a (point) light source. An area on the water surface is illuminated, but only a few light rays are reflected into the camera. Reflections are visible only if the surface normal takes a single, position-dependent value (see inset in figure 3). The slope $s=\tan \theta$, which is required for a reflection to be visible at a given image position with coordinates $u, v$, can be computed from the known positions of the light source and camera relative to the water surface. This 'visible' slope is a function of the image coordinates, as well as the surface distance, which can be obtained from processing of the stereo images.

If the light source were emitting in all directions, the range of slopes for which reflections are visible in the camera would only depend on the camera's field of view. In practice, the size of the footprint on the water surface that can be illuminated sufficiently by the light source is limited by the finite power of the source.

By averaging snapshots of reflections on the water surface, it is possible to measure the probability for slopes in a range of values to occur at the water surface (partial slope probability density function). For a static setup with an isotropic light source located at a distance from the water surface that can be considered constant, the mean brightness at a given image position corresponds to the mean probability to observe the specular slope, i.e. the slope for which the light source reflection is visible at this image position. In field experiments from a moving platform or research vessel, the platform's pitch and roll will add an offset and the varying distance to the water surface will affect the slope-position relation. In this case, it is necessary to have knowledge of the distance to the water surface to correctly process the images.

2.2.2. Estimation of the mean square slope. The wave slope probability density function (PDF) is known to be approximated by a Gaussian distribution (Cox and Munk 1954a, Bréon and Henriot 2006), as the higher moments caused by the non-linearity of the waves are small (Longuet-Higgins 1963)

The problem in estimating the mean square slope $\left(\sigma_{\mathrm{s}}^{2}\right)$ from the specular images comes from missing information. While the probability density function can be measured from analysing the distributions of reflections in the image, the range of measured slopes is determined by the field of view of the used camera. If only a narrow field of view is available, $\sigma_{\mathrm{s}}^{2}$ cannot be extracted directly from the distribution.

Therefore, an alternative approach is used here. Assuming that the slope PDF is Gaussian,

$$
p\left(s_{x}, s_{y}\right)=\frac{1}{2 \pi \sigma_{x} \sigma_{y}} \exp \left[-\left(\frac{s_{x}^{2}}{2 \sigma_{x}^{2}}+\frac{s_{y}^{2}}{2 \sigma_{y}^{2}}\right)\right],
$$

where $\sigma_{x}$ and $\sigma_{y}$ are the standard deviation of $s_{x}$ and $s_{y}$, respectively. The probability of slope zero is then

$$
p(0,0)=\frac{1}{2 \pi \sigma_{x} \sigma_{y}} .
$$




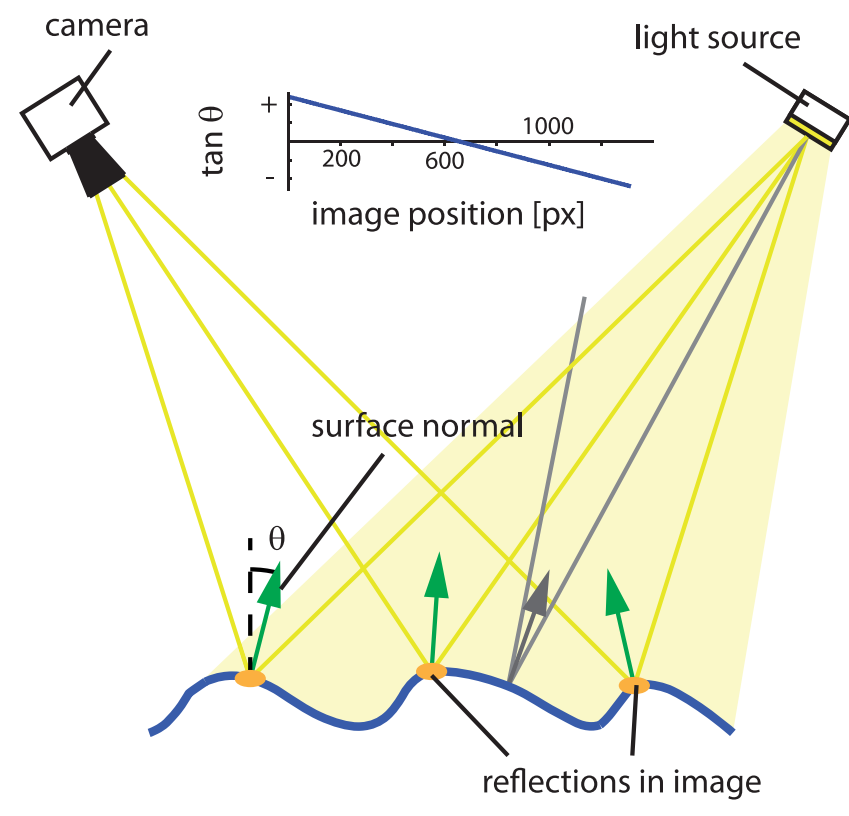

Figure 3. The principle of slope measurement: reflections are only visible if the water surface normal takes a specific, image positiondependent value.

Defining $\epsilon=\sigma_{x} / \sigma_{y}$, the product of the rms slope components can be rewritten as

$$
\sigma_{x} \sigma_{y}=\frac{1}{\epsilon} \sigma_{x}^{2} \quad \text { and } \quad \sigma_{x} \sigma_{y}=\epsilon \sigma_{y}^{2}
$$

With this, the mean square slope $\sigma_{\mathrm{s}}^{2}$ becomes

$$
\sigma_{\mathrm{s}}^{2}=\sigma_{x}^{2}+\sigma_{y}^{2}=\left(\epsilon+\frac{1}{\epsilon}\right) \sigma_{x} \sigma_{y} \propto \frac{(\epsilon+1 / \epsilon)}{p(0,0)} .
$$

Thus, the mean square slope $\sigma_{\mathrm{s}}^{2}$ is approximately inversely proportional to the probability of zero slope $p(0,0)$, as long as $(\epsilon+1 / \epsilon)$ is constant. This factor was found to be approximately constant over a wide range of conditions in several studies (see for details Kiefhaber 2010). The validity of this approximation is also investigated in the laboratory experiment described hereafter.

\subsection{Instrument}

The core of the RSSG, is shown in figure 4. The two cameras are mounted with a stereo base line of $b=300 \mathrm{~mm}$ and two line-shaped LED light sources are oriented orthogonal to the base line. Due to its small size with a short stereo base line, the instrument is very compact. This is helpful for creating a rugged design for easy deployment to field experiments. For high resolution stereo measurements, a high ratio of the base line to the working distance is beneficial. Kohlschütter (1906), Cote et al (1960), Holthuijsen (1983b) and Banner et al (1989), for example, all used ratios of about 0.5 (Jähne 1993). In more recent studies, Benetazzo (2006) used a ratio of 0.1, while Kosnik and Dulov (2011) used a ratio of 0.17 . Maintaining a sufficiently high resolution despite a decrease in the stereo base to working

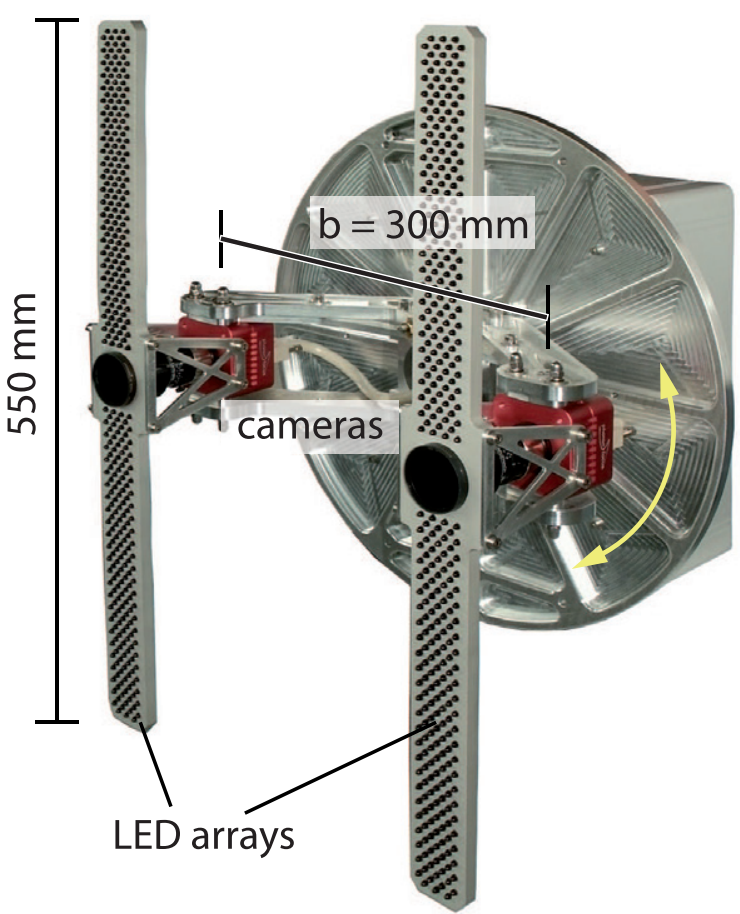

Figure 4. The core of the Reflective Stereo Slope Gauge (RSSG), consisting of two cameras and two line-shaped LED light sources.

distance ratio is possible due to advances in computer processing power and the possibility of determining the stereo disparity with sub-pixel accuracy. In our instrument, the ratio is 0.08 in the laboratory and 0.04 in field deployments. As we will show in section 3.2, this is sufficient to capture the dominant waves in both laboratory and field environments.

The cameras are Photonfocus MV1-D1312I-160-CL-12 1.4 mega pixel CMOS cameras with enhanced near-infrared sensitivity (quantum efficiency $>0.3$ at $950 \mathrm{~nm}$ ). They can acquire a maximum of 108 frames per second. In practice, 50 fps were usually used to limit the amount of raw data. The cameras are equipped with Schneider-Kreuznach TXR-2.2 $70 \mathrm{~mm}$ lenses, providing a field of view of $8.4^{\circ} \times 6.9^{\circ}$. This narrow field of view was chosen since no wide-angle LEDs with sufficiently high power were available for the light sources at the time the instrument was built (in 2010). The range of slopes for which the slope PDF can be measured is about $[-0.07,0.07]$. This range is too small to directly extract $\sigma_{\mathrm{s}}^{2}$ from the distribution width, so that this quantity is estimated from the probability of zero slope $p(0)$.

The light sources consist of $2 \times 350$ narrow-angle nearinfrared LEDs (OSRAM SFH-4545, $\lambda_{\text {peak }}=950 \mathrm{~nm}, 100$ $\mathrm{mA}$ current rating). This wavelength is closely matched to a major water absorption peak (at $970 \mathrm{~nm}$ ). Therefore, light that is not reflected at the water surface $(98 \%$ of the incident light) is rapidly absorbed by the water body. The low penetration depth of only $3.5 \mathrm{~cm}$ suppresses upwelling light that is reflected off particles or objects floating in the water. In addition, interference bandpass filters on the cameras limit their sensitivity to the spectral band of the light sources $(950 \pm 25 \mathrm{~nm})$. 
a

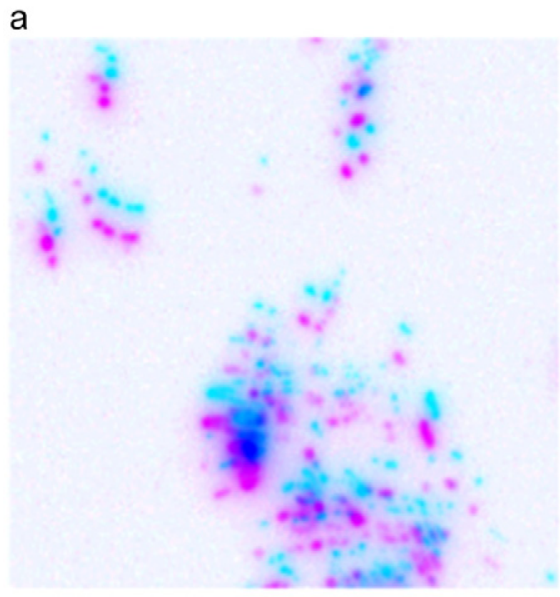

b

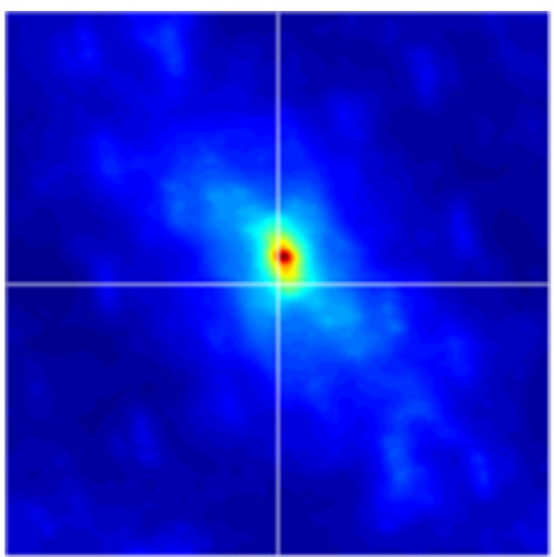

Figure 5. (a) Detail from a typical RSSG stereo image containing multiple reflections. The image is inverted, so that the background is white. The left camera image is shown in magenta, the right in cyan. (b) Computed cross correlation.

As was noted in section 2.1.1, it is required that each camera only observes light from the opposing light source. This is achieved by sequential image acquisition. The exposure is set to $0.2 \mathrm{~ms}$ and the exposure of the second camera is delayed by $0.2 \mathrm{~ms}$. The water surface does not change significantly over this short period. The light sources are flashed only for the exposure of the cameras. Due to the low dutycycle and short pulse duration, it is possible to overdrive each LED with a current of $1 \mathrm{~A}$ (instead of $100 \mathrm{~mA}$ ).

Due to their elongated shape, the light sources provide sufficient light output, but can still be considered pointlike in the camera baseline direction. For these linear light sources, the reflections that are visible in the two images no longer exactly come from the same locations on the water surface, as was assumed in figure 1 and in the derivation of (1). The error associated with this is estimated in the appendix. Under all realistic conditions, the error in the reconstructed surface height is well below $1 \mathrm{~mm}$. As the goal of the height measurement is to measure the height of the dominant waves, not of the smallest ripples on the surface, this is acceptable.

The effect on the slope measurement can be described mathematically as a convolution of the slope PDF with a light source function. When viewed from the water surface in $3.5 \mathrm{~m}$ distance, the long side of the light source appears under an angle of $\pm \tan \theta=550 \mathrm{~mm} /(2 \times 3500 \mathrm{~mm})=0.078$, i.e. an angle of $\pm 5^{\circ}$. An effect on the slope PDF is only observed if the rms surface slope is in the same range, which requires a very smooth water surface. In field measurements, the working distance is typically greater than $8 \mathrm{~m}$ so the disturbance is even smaller.

\subsection{Data processing}

2.4.1. Mean square slope. A number of effects other than the shape of the water surface influence the appearance of light source reflections in the images. First of all, to allow measurements under a wide range of wind speeds and surface roughness, the light source brightness can be varied by more than an order of magnitude. The distance $R$ of the water surface also impacts the reflection brightness, which follows a $1 / R^{2}$ law. Thirdly, imperfections of the imaging system (vignetting, geometrical shading and image sensor non-uniformities) and the light sources' directional characteristics create spatial inhomogeneities in the brightness distribution.

The effects of the imperfections of the imaging system are removed with a two point radiometric calibration (Jähne 2005). The light sources have been extensively characterised. The dependence of brightness on current and emission angle were determined and corrected (for details, see Kiefhaber 2014). In this laboratory study, the typical wave heights were less than $1 \%$ of the distance between the instrument and the water surface. Therefore, corrections for the $1 / R^{2}$ dependence were not necessary. Also, for such small changes in the distance of the water surface to the instrument, the relation between the image position and 'visible' slope can be considered constant. As an example, for a change in water surface distance of $\pm 5 \mathrm{~cm}$, the slope that is visible in the centre of the image changes by only $\Delta s= \pm 6 \times 10^{-4}$.

After applying these corrections, the calibrated images are averaged in time to obtain the truncated slope PDF. The probability for slope 0 is then estimated by taking the mean of the grey values in a window of $150 \times 150$ pixels around the centre of the image. This corresponds to averaging the probability of the slopes in an interval of $\pm 0.5^{\circ}$ (slope \pm 0.009). The RSSG surface roughness parameter $\chi$ is thus

$$
\chi=\left(\left(\mathrm{N}_{x} \cdot \mathrm{N}_{y}\right)^{-1} \sum_{-\mathrm{N}_{x} / 2}^{\mathrm{N}_{x} / 2} \sum_{-\mathrm{N}_{y} / 2}^{\mathrm{N}_{y} / 2} I(x, y)\right)^{-1},
$$

where $\mathrm{N}_{x}=150$ and $\mathrm{N}_{y}=150$ and $I(x, y)$ is the calibrated mean image, which is equivalent to the (unnormalised) slope PDF.

2.4.2. Surface displacement. A detail of a typical RSSG image is shown in figure $5(\mathrm{a})$. The cross correlation $(\mathrm{CC})$ of 


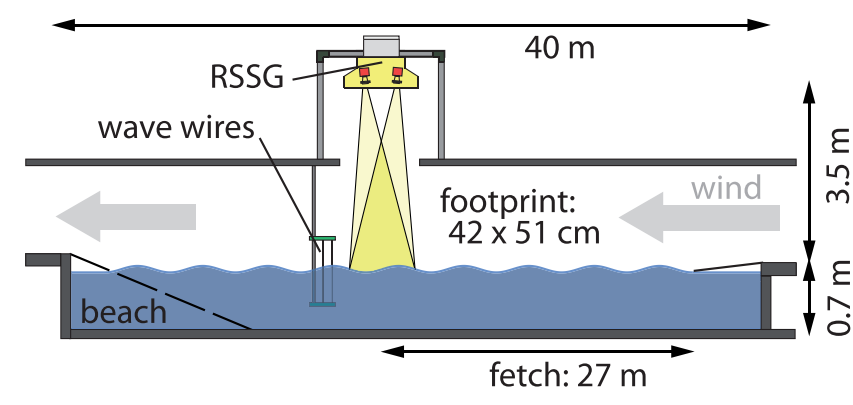

Figure 6. The experimental setup at the Pythéas facility in Marseille-Luminy.

the two images is computed (see figure 5(b)); the position of the maximum is equal to the stereo disparity.

The CC is computed in Fourier space, where it reduces to

$$
C C(\mathbf{x})=\int_{-\infty}^{+\infty} \int \mathrm{d} \mathbf{x} g_{1}^{*}(\mathbf{x}) g_{2}\left(\mathbf{x}^{\prime}+\mathbf{x}\right)=\mathcal{F}^{-1}\left[\hat{g}_{1}^{*}(\mathbf{k}) \hat{g}_{2}(\mathbf{k})\right],
$$

where $\mathcal{F}^{-1}$ is the inverse Fourier transform (iFT) and $\hat{g}_{1}(\mathbf{k})$ and $\hat{g}_{2}(\mathbf{k})$ are the Fourier transforms (FT) of the left and right interrogation windows, respectively. Since we know that the maximum of the $\mathrm{CC}$ is moving only along the $y$-axis (the direction of the stereo base), it is sufficient to compute the CC for a couple of columns. Instead of using standard FFT algorithms, we compute the iFT directly by matrix multiplication (for details, see Kiefhaber 2014). Since this approach allows us to compute the $\mathrm{CC}$ only in the few columns we need, it results in a significant speed-up.

In Fourier space, it is also straightforward to compute the cross correlation with subpixel accuracy. This is typically done by 'zero padding', i.e. embedding the complex product $\hat{g}_{1}^{*}(\mathbf{k}) \hat{g}_{2}(\mathbf{k})$ into a larger array of zeros before applying the iFT. The computational cost of this procedure scales with $u^{2}$, where $u$ is the upsampling factor. We therefore implemented the algorithm proposed by Guizar-Sicairos et al (2008), where FT by matrix multiplication is used to compute the upscaled $\mathrm{CC}$ around the peak only (factor $u=10$ ).

\subsection{Laboratory validation study}

A laboratory validation experiment to demonstrate the capabilities of the RSSG was conducted in March 2011 in the large air-sea interaction facility of the Institute de Recherche sur les Phenomènes Hors Equilibre (IRPHE) ${ }^{2}$ in Marseille-Luminy. The facility consists of a $40 \mathrm{~m}$ long and $2.6 \mathrm{~m}$ wide water tank. The water depth is $0.9 \mathrm{~m}$, the air space is $1.6 \mathrm{~m}$ high. The RSSG was set up $27 \mathrm{~m}$ downwind of the beginning of the tank and mounted at a distance of $3.6 \mathrm{~m}$ from the water surface looking down vertically through a small hole in the otherwise closed facility, see figure 6. A detailed description of the facility is given by Coantic and Bonmarin (1975).

Waves were generated by wind only in a range of wind speeds between 2 and $13 \mathrm{~m} \mathrm{~s}^{-1}$. The water surface was cleaned each day before the measurements. Two capacitance-type

\footnotetext{
2 The facility is now affiliated with the Institute Pythéas in Marseille.
}

wire wave gauges sampled the water surface elevation at a frequency of $250 \mathrm{~Hz}$ for a wave height reference. The gauges were installed just downwind of the RSSG's field of view.

Measurements for surface roughness statistics were performed on two consecutive days, 9 March 2011 and 10 March 2011. On the last day of the experiment, 11 March 2011, the wave gauges were moved into the RSSG's field of view for a direct comparison with the RSSG's height measurement.

An overview of the physical and technical specifications used in the experiments is presented in table 1. For each run, the reference wind speed $\left(U_{\text {ref }}\right)$, the used LED brightness setting (LED pulse current), the length of the individual image sequences (seq.) and the total amount of data recorded (tot. data) are given, as well as the time duration (dur.) over which the (non-continuous) sampling was distributed. The results for the RSSG roughness parameter $\chi$ will be discussed in section 3 and compared to the reference mean square slope values given in the last column of the table. The latter were measured in an earlier experiment with a laser slope gauge (LSG), a well-established technique for measuring small-scale wave slopes. This technique is based on the measurement of the refraction angle of a vertically-looking laser beam after crossing the air-water interface (e.g. Lange et al 1982).

\section{Results and discussion}

\subsection{Surface roughness}

The appearance of the water surface changes drastically over the range of wind speeds investigated in the experiments $\left(2-13 \mathrm{~m} \mathrm{~s}^{-1}\right)$. This is clearly seen in the raw images (captured by one of the cameras) of the RSSG shown in figure 7 . The increasing surface roughness leads to a decreasing reflection pattern size and brightness. The displayed area of the water surface is about $30 \mathrm{~cm} \times 20 \mathrm{~cm}$. At low wind speeds, reflections appear as more or less closed large loops with wide gaps in between. These are contour lines of alongwind slope zero (Cox and Zhang 2011). At higher wind speeds, the reflections are more point-like and nearly uniformly distributed over the water surface. The main reason for the change in appearance is the increasing curvature of the water surface.

The surface roughness parameter $\chi$, which is ideally proportional to the mean square slope $\sigma_{\mathrm{s}}^{2}$, is computed from the images as described in section 2.4.1. In figure 8, $\chi$ is plotted against the reference mean square slope values obtained previously with the LSG. The variation with wind speed of both quantities over the full range of measurement conditions is undoubtedly well correlated, $\chi$ exhibiting a linear dependence on $\sigma_{\mathrm{s}}^{2}$. Different symbols are used to distinguish the data obtained on two different days, as well as from the images of the two RSSG cameras (left and right). The good agreement of the measurement results from 9 and 10 March demonstrates the repeatability of roughness conditions in the facility. Thus, we expect that using the LSG results from another experiment for comparison is justified. 
Table 1. Overview of the measurement runs and obtained results for the surface roughness (see section 3 ).

\begin{tabular}{|c|c|c|c|c|c|c|c|c|}
\hline$U_{\text {ref }}\left(\mathrm{m} \mathrm{s}^{-1}\right)$ & Date & run & $\mathrm{LED}(\mathrm{mA})$ & Seq. (s) & tot. data (s) & dur. (min) & $\chi(\mathrm{RSSG})$ & $\sigma_{\mathrm{s}}^{2} \times 100(\mathrm{LSG})$ \\
\hline \multirow[t]{2}{*}{2} & $09 / 03$ & run $1 b$ & 100 & 1 & 401 & 30 & 0.073 & 0.828 \\
\hline & $10 / 03$ & run1 & 100 & 1 & 201 & 18 & 0.057 & \\
\hline \multirow[t]{2}{*}{3} & 09/03 & run2 & 230 & 1 & 402 & 32 & 0.571 & 1.392 \\
\hline & $10 / 03$ & run2 & 230 & 1 & 301 & 27 & 0.554 & \\
\hline \multirow[t]{2}{*}{4} & $09 / 03$ & run3 & 250 & 1 & 906 & 73 & 0.818 & 1.954 \\
\hline & $10 / 03$ & run3 & 250 & 1 & 300 & 27 & 0.823 & \\
\hline 5 & $10 / 03$ & run4 & 260 & 1 & 800 & 72 & 1.010 & 2.606 \\
\hline \multirow[t]{2}{*}{6} & $09 / 03$ & run4 & 280 & 1 & 436 & 35 & 1.343 & 3.641 \\
\hline & $10 / 03$ & run5 & 280 & 1 & 300 & 27 & 1.312 & \\
\hline \multirow[t]{2}{*}{7} & 09/03 & run5 & 280 & 1 & 505 & 55 & 1.939 & 4.263 \\
\hline & $11 / 03$ & mitDraht4,5 & 280 & 8 & 15 & 1 & & \\
\hline \multirow[t]{2}{*}{8} & $09 / 03$ & run6 & 325 & 1 & 405 & 32 & 2.266 & 5.440 \\
\hline & $10 / 03$ & run6 & 325 & 1 & 610 & 55 & 2.398 & \\
\hline \multirow[t]{2}{*}{9} & 09/03 & run7 & 350 & 1 & 314 & 25 & 2.720 & 6.727 \\
\hline & $10 / 03$ & run7 & 350 & 1 & 400 & 36 & 2.849 & \\
\hline \multirow[t]{2}{*}{10} & 09/03 & run8 & 420 & 1 & 808 & 65 & 2.911 & 7.593 \\
\hline & $11 / 03$ & long9 & 420 & 19 & 56 & 7 & & \\
\hline \multirow[t]{2}{*}{11} & $10 / 03$ & run $8 b$ & 500 & 1 & 700 & 65 & 3.234 & 8.948 \\
\hline & $11 / 03$ & mitDraht1,2,3 & 500 & 8 & 23 & 1 & & \\
\hline \multirow[t]{2}{*}{12} & $09 / 03$ & run9 & 1000 & 1 & 617 & 50 & 4.476 & 10.190 \\
\hline & $10 / 03$ & run9 & 1000 & 1 & 505 & 47 & 4.550 & \\
\hline \multirow[t]{2}{*}{13} & 09/03 & run10 & 1000 & 1 & 712 & 62 & 4.737 & 10.740 \\
\hline & $10 / 03$ & run 10 & 1000 & 1 & 515 & 51 & 4.836 & \\
\hline
\end{tabular}

Note: The reference mean square slope values in the last column were recorded with an LSG in an earlier experiment.
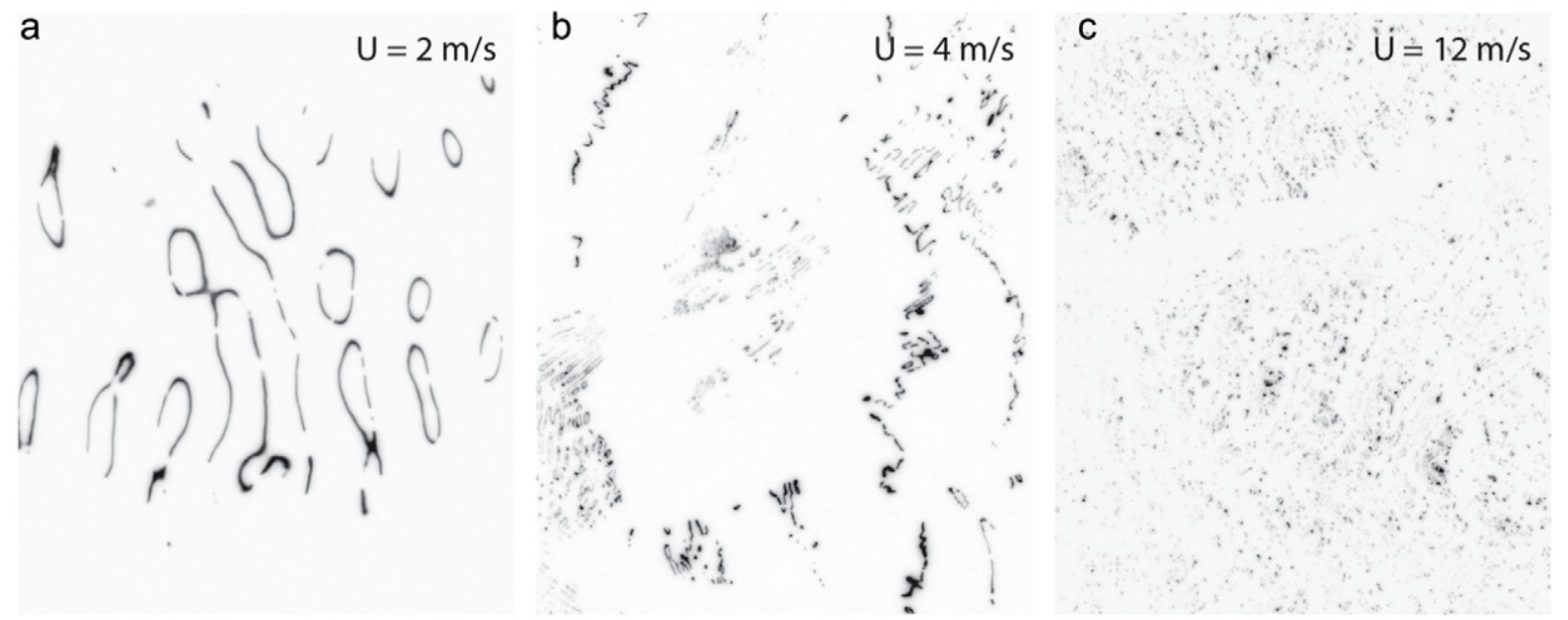

Figure 7. Three details from RSSG images at different wind speeds displayed with inverted grey scale (the black areas are brightest in the original images, white is the background) and contrast-enhanced for visibility. The wind is blowing from left to right with speeds of (a) $2 \mathrm{~ms}^{-1}$, (b) $4 \mathrm{~ms}^{-1}$ and (c) $12 \mathrm{~ms}^{-1}$.

There is one significant deviation in the $\chi$ measurements from the linear fit, at $\sigma_{\mathrm{s}}^{2}=0.09$. Unfortunately, this condition was only measured once over the two main measurement days. The deviation cannot be explained by statistical errors, as the measurement time for the run at this wind speed (run8 at $10 \mathrm{~m} \mathrm{~s}^{-1}$, see table 1) is among the longest ones of the experiment. In fact, we have a small data set recorded at the same wind speed on the third measurement day (11 March, square symbol), which the $\chi$ measurement fits well with the data obtained at the other wind speeds. Since this measurement technique provides consistent results for all conditions except the 9 March run8, we expect the outlier to be due to a faulty record of the setting of the light source brightness or the wind speed in the facility. The statistical error of the measurement can be estimated from the standard deviation of running $\chi$ means. It is found to be smaller than $1 \%$ for averaging windows longer than $100 \mathrm{~s}$, which is well fulfilled for all the conditions presented here. The error increases for shorter averaging intervals, but remains below $5 \%$ for time intervals longer than $20 \mathrm{~s}$.

\subsection{Surface displacement}

The accuracy of the RSSG water surface elevation measurements was investigated by comparing them with those obtained 


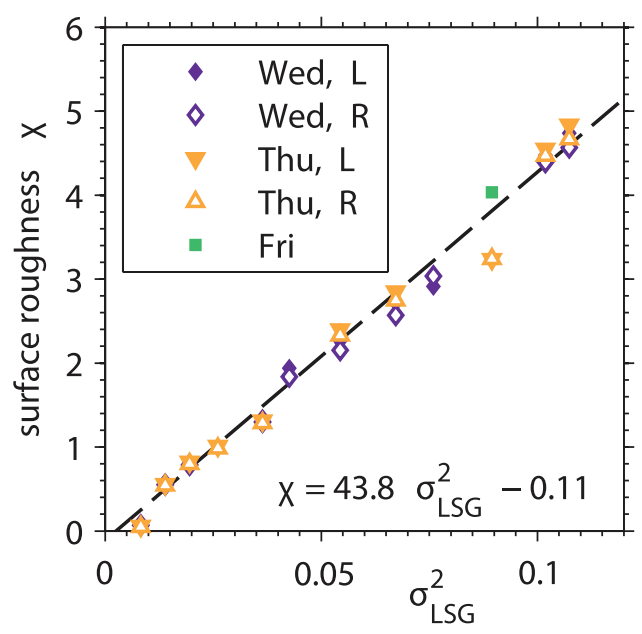

Figure 8. Comparison of the RSSG surface roughness parameter $\chi$ with reference $\sigma_{\mathrm{s}}^{2}$ measurements by an LSG. The measurements agree well over the full range of conditions.

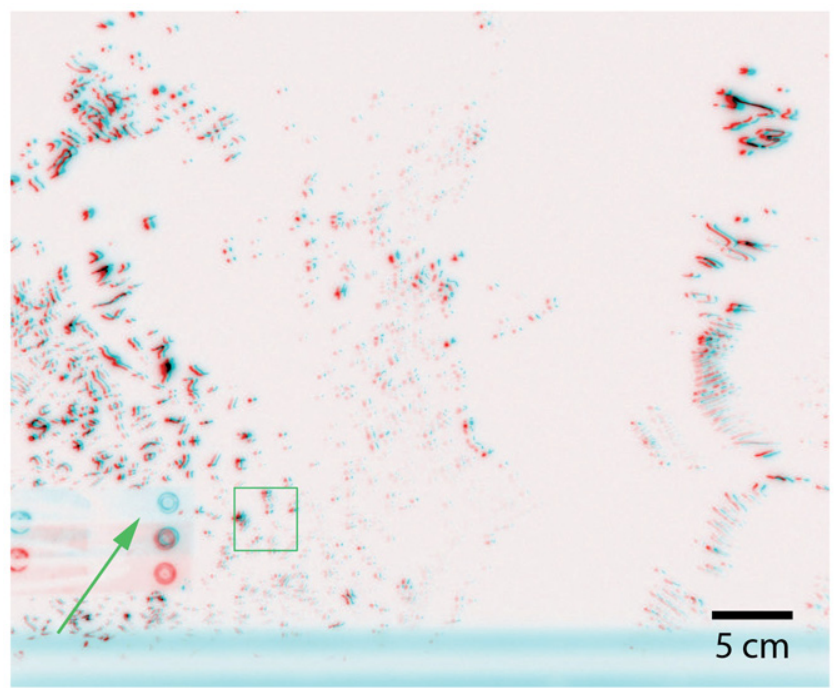

Figure 9. False-colour stereo image of the RSSG, the left camera is shown in red, the right camera in cyan. In the lower left corner, the mount of the wave wire is visible; the wire is below the green arrow. The green square is the interrogation window used for the comparison.

from the capacitance-type wave probe. To get a point-like measurement of water height, a small interrogation window of $100 \times 100$ pixels (about $4 \times 4 \mathrm{~cm}$ footprint) was used; the window was placed just upwind of the wave wire gauge, see figure 9 (the wave probe is located underneath the tip of the green arrow). A smaller window was found to be impractical, since data dropouts (missing data points due to a lack of light source reflections in the window) increased dramatically. The number of image pairs for which the water surface elevation could be determined depends strongly on the availability of the surface roughness of scales smaller than the interrogation window. As already seen in figure 7, the images of the water surface observed at higher wind speeds generally exhibit more evenly distributed reflection patterns.

Time series of the wave signals obtained by both measurement techniques are compared for a very rough surface

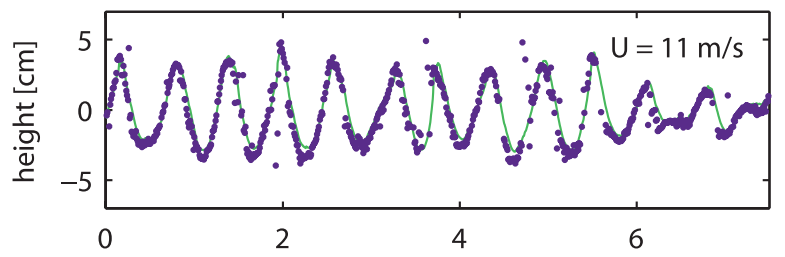

b

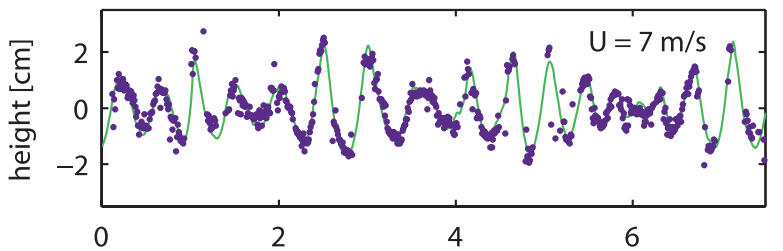

C

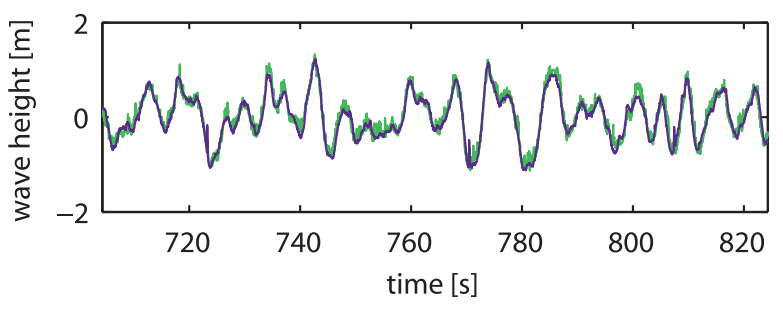

Figure 10. (a) Comparison of wave height measurements of the RSSG (purple dots) with a nearly co-located wave wire (green line) at a wind speed of $11 \mathrm{~m} \mathrm{~s}^{-1}$ in the Marseille facility. (b) Same for wind speed of $7 \mathrm{~m} \mathrm{~s}^{-1}$. (c) Field comparison with measurements by a Riegl laser altimeter in the tropical Pacific. Note the different scale in time and height.

condition (at $U_{\text {ref }}=11 \mathrm{~m} \mathrm{~s}^{-1}$ ) in figure 10 (a). The RSSG data was shifted in time to account for the upwind location of the measurement, as the change in the wave shape should not change significantly over the short distance between both measurements. The agreement between the RSSG (dots) and wave gauge (solid line) signals is generally good, the deviations are limited to a few millimetres. Figure 10(b) shows wave signals obtained at a lower wind speed $\left(U_{\text {ref }}=7 \mathrm{~m} \mathrm{~s}^{-1}\right)$, where the reflecting water surface patches are less evenly distributed. We notice a sharp increase in the occurrence of data dropouts. However, as both wave crests and troughs are characterised by points of near zero slope, their heights are generally measured correctly, allowing for a reconstruction of the wave profile by using an interpolation method. In these cases, we adopted a piecewise cubic Hermite polynomial interpolation method, as suggested by Veron et al (2009) for similar datasets.

The lower panel in figure 10(c) shows measurements performed at sea from R/V Kilo Moana in the tropical Pacific during the KM1130 cruise. The RSSG data are shown in purple, while the reference wave height measurements plotted in green were provided by a Riegl laser altimeter. This instrument is based on measuring the return time of laser pulses reflected from the water surface. Both the RSSG and Riegl measurements can be affected by data dropouts, as both instruments rely on the reflection of light. However, owing to its much higher acquisition rate $(2000 \mathrm{~Hz})$, long wave profiles reconstructed from the Riegl altimeter data set seem less affected. The two wave time series agree very well (note the different time and height scales compared to the laboratory measurements). Note, however, that the signal derived from the Riegl altimeter is a little more noisy due to both the sensor's capability 


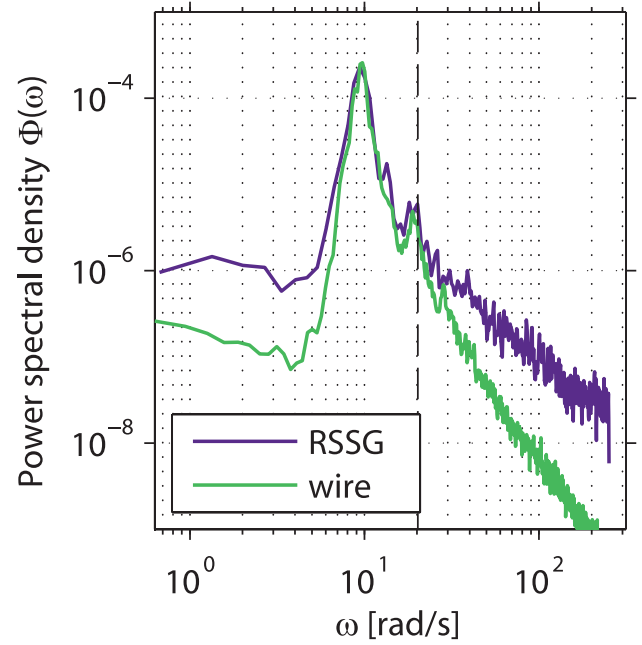

Figure 11. Wave height power spectral density computed from RSSG (purple) and wave wire measurements (green). The measurements agree well around the dominant wave peak.

of resolving smaller scale waves than the RSSG imaging technique and its measurement resolution uncertainty $(\Delta h=2 \mathrm{~cm}$, according to the manufacturer). Here in particular, the RSSG signal looks smoother due to the choice of a relatively large interrogation window of $200 \times 200$ pixels. At the shipborne working distance of $9 \mathrm{~m}$, this corresponds to a footprint of $20 \times 20 \mathrm{~cm}$ at the water surface over which the wave height is averaged. Thus, information about small-scale waves is lost.

The effect of the finite size of the interrogation window can be studied more quantitatively in the laboratory with spectral analysis, which is commonly used in oceanography to describe wave fields. Figure 11 displays the power spectra computed from the wave time series measured by the wave gauge and RSSG in green and purple, respectively. Both spectra fit very well at the dominant wave peak around $10 \mathrm{rad} \mathrm{s}^{-1}$. However, compared to the reference spectrum estimated from the wave probe signal, the RSSG wave spectrum overestimates the spectral energy at low and at high frequencies. At low frequencies, this very likely results from the short sampling time of the RSSG, leading to non-zero time averages for the lowest frequency components.

At high frequencies, the deviation is caused by the noise introduced by the finite size of the interrogation window $(200 \times 200$ pixels, equivalent to $8 \times 8 \mathrm{~cm})$ and the absence of filtering at the corresponding spatial Nyquist frequency. To estimate the spatial scale resolution of the instrument, we can use a Nyquistlike criterion, assuming that the shortest wavelength to be determined should be at least twice as long as the image size. This gives a cut-off wavenumber of $k_{\mathrm{c}}$ of order of $k_{\mathrm{c}}=2 \pi / \lambda=39.3 \mathrm{rad} \mathrm{m}^{-1}$. According to the linear dispersion relation for water waves (Kundu 2008), this corresponds to a frequency of $\omega=19.7 \mathrm{rad} \mathrm{s}^{-1}$, which is marked by the dashed line in figure 11. This cut-off frequency corresponds well with the onset of the overestimation of energy in the RSSG wave spectrum.

\subsection{Discussion}

Compared to well-established wave height and wave slope measurement techniques, e.g. wave wire probes and the LSG, the optical and reflection-based measuring methods used in the RSSG are rather indirect. Therefore, to test the validity of these new measurement concepts and to compare the results obtained to reference measurements for evaluating the accuracy of the instrument is a crucial step. This was achieved in this work and we were able to show that both the surface roughness and wave height measurements agree well with the equivalent reference quantities. To use the RSSG does not mean to replace wave wire gauges, and better devices exist for estimating wave slope statistics in the laboratory, e.g. the imaging slope gauge (Kiefhaber et al 2014). All of these instruments, however, require parts both above and below the water surface. The RSSG is based solely on the reflection of light at the water surface, making it much easier to deploy to shipborne field experiments. Also, there is no interference with waves or currents.

The surface roughness parameter $\chi$ that is obtained from RSSG measurements was shown to be proportional to the mean square slope $\sigma_{\mathrm{s}}^{2}$. Quantitative measurements of smallscale surface roughness are not readily available from existing instruments, but help in improving our understanding of airsea interaction processes. The statistical error of the measurement is small. It is found to be less than 5\% for averaging intervals of $20 \mathrm{~s}$ and more. This allows for local measurements at high temporal resolution. On the other hand, to obtain a meaningful parameter for the general state of the sea surface, longer sampling intervals will generally be required to account for low frequency periodicities, e.g. modulations of small-scale waves by swell groups.

To obtain absolute values of $\sigma_{\mathrm{s}}^{2}$ from the measured $\chi$, the system needs to be carefully calibrated. The linear relation of $\chi$ to $\sigma_{\mathrm{s}}^{2}$ given in section 8 can be used, if the changing distance of the instrument to the water surface under open ocean conditions is accounted for. With more recently developed high-power LEDs, it is also possible to illuminate a larger footprint on the water surface and use a camera with a larger field of view. Thus, it is possible to measure the slope PDF directly up to larger maximum slopes. Then, $\sigma_{\mathrm{s}}^{2}$ can be determined directly as the variance of the PDF.

The comparison of the RSSG water height measurements with a capacitance-type wave wire demonstrates that the RSSG is capable of measuring the distance to the water surface accurately with errors of the order of a few millimetres. In laboratory studies with wave amplitudes of a few centimetres this may be a large relative error. The real strength of the RSSG is its capability of measuring with a similar precision in field studies, when the overall wave amplitude is of the order of meters. The measurement precision is mostly limited by the required finite size of the interrogation window. While smaller window sizes allow one to resolve smaller scale waves, they also lead to increased data dropout, as the probability of not measuring any reflections in the window increases. To estimate the peak wave frequency and significant wave height on the other hand, important parameters in the description of large scale waves, small windows are not required, so that they can be extracted reliably from the RSSG data. 


\section{Conclusion}

A new optical instrument for the measurement of ocean wave parameters in field studies, the Reflective Stereo Slope Gauge (RSSG) was presented. The performance of the RSSG was tested under controlled conditions in a laboratory facility. It was verified that the RSSG surface roughness parameter $\chi$, derived from the probability of zero slope of the surface, is proportional to the mean square slope $\sigma_{\mathrm{s}}^{2}$ for a large range of conditions. The height measurement from stereo triangulation using a dedicated setup of cameras and light sources was shown to measure water height with high precision with errors of the order of a few millimetres. The combination of these capabilities with the insensitivity to environmental conditions, the instrument's capability of measuring day and night, and the ease of deployment in field studies due to the reflection-based measurement technique make the RSSG a valuable addition to the spectrum of available wave measurement techniques.

\section{Acknowledgments}

Partial financial support by the German Research Foundation (DFG, grant number Ja395/15-1), as well as the German Federal Ministry of Education and Research (BMBF) joint project 'Surface Ocean Processes in the Anthropocene' (SOPRAN, FKZ 03F0662F and FKZ 03F0462F) are gratefully acknowledged.

\section{Appendix. Effect of the size of the light sources}

In figure 1, an idealised setup was shown in which two light sources were located virtually at the same location as two cameras. However, in the instrument (see figure 4), two line-shaped light sources were used. While in principle this geometry causes an error in the height measurement (Cox and Zhang 2011), for our setup this error is negligible, as will be detailed below.

We define a coordinate system such that the $x$-axis is aligned with the stereo base and the $y$-axis orthogonal to that, parallel to the light sources. The $z$-axis is forward, towards the water surface. In such a coordinate system, the positions of the cameras are

$$
\mathbf{c}_{\mathrm{L}}=\left(\begin{array}{c}
-b / 2 \\
0 \\
0
\end{array}\right), \quad \text { and } \quad \mathbf{c}_{\mathrm{R}}=\left(\begin{array}{c}
b / 2 \\
0 \\
0
\end{array}\right),
$$

with the stereo baseline $b$. The question that needs to be answered is: given a position on the water surface $\mathbf{r}=\left(r_{x}, r_{y}, r_{z}\right)$, what is the surface slope $\mathbf{n}_{\mathrm{L}}$ that is required at this position, so the left camera sees the reflection of the right light source and what is the surface slope $\mathbf{n}_{R}$ that is required so the right camera see the reflection of the left light source?

Assume two light rays originating from the left and right light sources in position

$$
\mathbf{o}_{\mathrm{L}}=\left(\begin{array}{c}
-b / 2 \\
l \\
0
\end{array}\right), \quad \text { and } \quad \mathbf{o}_{\mathrm{R}}=\left(\begin{array}{c}
b / 2 \\
l \\
0
\end{array}\right) \text {, }
$$

where $l$ is the position along the linear light source. At point $\mathbf{r}$ on the water surface, light emitted from point $\mathbf{o}_{\mathrm{L}}$ is reflected into the right camera at $\mathbf{c}_{\mathrm{R}}$ if

$$
\begin{gathered}
\mathbf{n}_{\mathrm{R}}=\frac{\mathbf{o}_{\mathrm{L}}-\mathbf{r}}{\left|\mathbf{c}_{\mathrm{R}}-\mathbf{r}\right|} \\
=\left(\begin{array}{c}
\frac{-b / 2-r_{x}}{\sqrt{\left(b / 2+r_{x}\right)^{2}+\left(l-r_{y}\right)^{2}+r_{z}^{2}}}+\frac{b / 2-r_{x}}{\sqrt{\left(b / 2-r_{x}\right)^{2}+r_{y}^{2}+r_{z}^{2}}} \\
\frac{l-r_{y}}{\sqrt{\left(b / 2+r_{x}\right)^{2}+\left(l-r_{y}\right)^{2}+r_{z}^{2}}}-\frac{r_{y}}{\sqrt{\left(b / 2-r_{x}\right)^{2}+r_{y}^{2}+r_{z}^{2}}} \\
-\frac{r_{z}}{\sqrt{\left(b / 2+r_{x}\right)^{2}+\left(l-r_{y}\right)^{2}+r_{z}^{2}}}-\frac{r_{z}}{\sqrt{\left(b / 2-r_{x}\right)^{2}+r_{y}^{2}+r_{z}^{2}}}
\end{array}\right) .
\end{gathered}
$$

A similar, but not identical, expression is obtained for the opposite case, light emitted from point $\mathbf{o}_{\mathrm{R}}$ is reflected into the left camera at $\mathbf{c}_{\mathrm{R}}$ at point $\mathbf{r}$ if

$$
\mathbf{n}_{\mathrm{L}}=\frac{\mathbf{o}_{\mathrm{R}}-\mathbf{r}}{\left|\mathbf{c}_{\mathrm{L}}-\mathbf{r}\right|}
$$

$$
=\left(\begin{array}{c}
\frac{b / 2-r_{x}}{\sqrt{\left(b / 2-r_{x}\right)^{2}+\left(l-r_{y}\right)^{2}+r_{z}^{2}}}+\frac{-b / 2-r_{x}}{\sqrt{\left(b / 2+r_{x}\right)^{2}+r_{y}^{2}+r_{z}^{2}}} \\
\frac{l-r_{y}}{\sqrt{\left(b / 2-r_{x}\right)^{2}+\left(l-r_{y}\right)^{2}+r_{z}^{2}}}-\frac{r_{y}}{\sqrt{\left(b / 2+r_{x}\right)^{2}+r_{y}^{2}+r_{z}^{2}}} \\
-\frac{r_{z}}{\sqrt{\left(b / 2-r_{x}\right)^{2}+\left(l-r_{y}\right)^{2}+r_{z}^{2}}}-\frac{r_{z}}{\sqrt{\left(b / 2+r_{x}\right)^{2}+r_{y}^{2}+r_{z}^{2}}}
\end{array}\right) .
$$

Before analysing the error for the general case, an important special case is considered: if the point of reflection is at $\mathbf{r}=\left(0,0, r_{z}\right)$, both surface normal vectors are identical if $l^{2} \ll\left(r_{z}^{2}+b^{2} / 4\right)$. Thus, if the water surface is sufficiently far away (compared to the size of the light source $l$ ) and a point near $\mathbf{r}=\left(0,0, r_{z}\right)$ is observed, both cameras will see reflections of their respective light sources from the same spot on the surface.

In figure $\mathrm{A} 1$, the norm of the difference between surface normals $\mathbf{n}_{\mathrm{L}}$ and $\mathbf{n}_{\mathrm{R}}$ is shown for the geometry of the laboratory experiment. The difference is shown for different positions on the water surface $r_{x}, r_{y}$ at a fixed distance $r_{z}=3500 \mathrm{~mm}$. The shown surface patch corresponds to the footprint of the RSSG. Figure A1(a) shows the error caused by an emitter placed at the end of the RSSG light sources, while figure $\mathrm{A} 1(\mathrm{~b})$ is the average error for the linear light sources. The rms difference between the surface normal causing a reflection into the left camera and the normal causing a reflection into the right camera is $\Delta s=9.4 \times 10^{-5}$.

Propagating this difference into an error in the water elevation measurement is not straightforward and requires additional assumptions. However, in any realistic case the error is small. The error strongly depends on surface curvature, as curvature determines how large the distance between two positions with the slope difference $\Delta s$ is. An error in height of $\Delta h=1 \mathrm{~mm}$ translates to an error of $\Delta d=0.22 \mathrm{px}$ in disparity in the laboratory setting, according to (1). This is equivalent to a position difference on the water surface of 

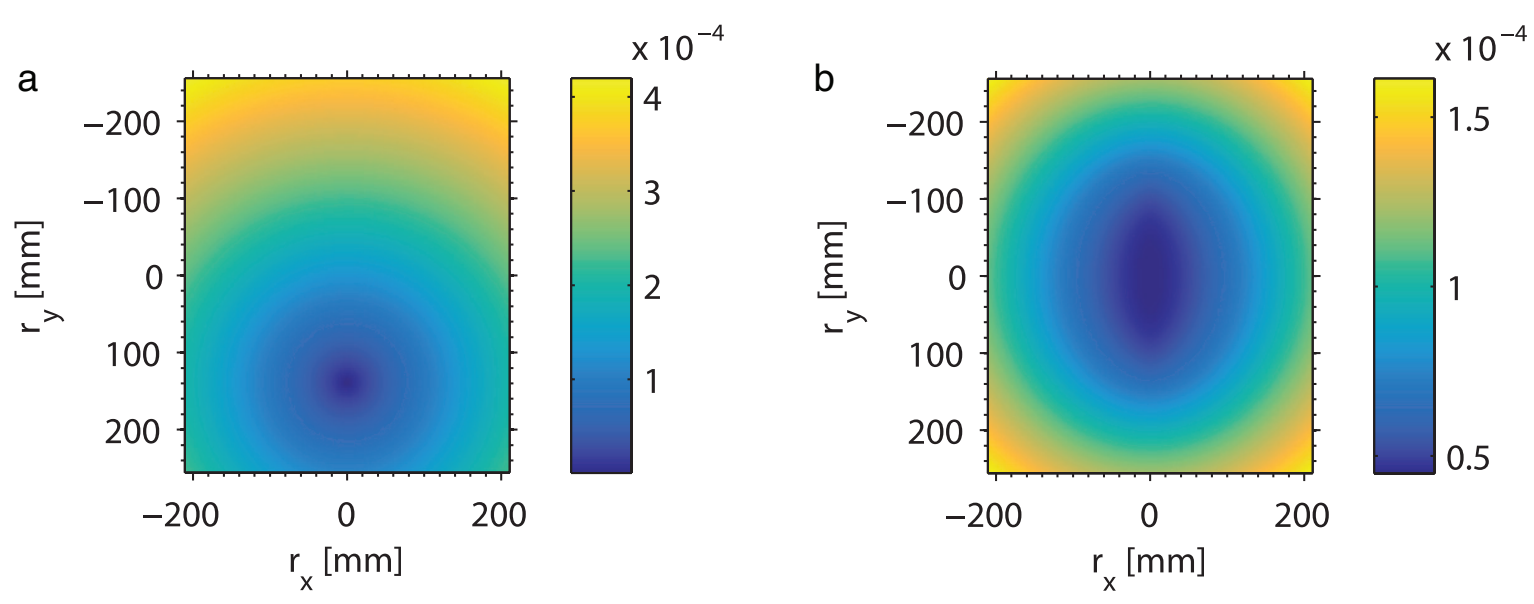

Figure A1. (a) Slope error for a single emitter, placed at $l=275 \mathrm{~mm}$. (b) Average slope error for emitters along a line from $l=275 \mathrm{~mm}$ to $l=-275 \mathrm{~mm}$.

$$
\Delta x=\frac{\Delta \mathrm{dZ}_{0}}{0.008 \mathrm{~mm} \mathrm{px}^{-1} \cdot f}=0.086 \mathrm{~mm},
$$

with the focal length $f=72 \mathrm{~mm}$ and the mean distance to the water surface $\mathrm{Z}_{0}=3500 \mathrm{~mm}$. The minimum curvature $c$ and the maximum curvature radius $R$ for which the slope changes by $\Delta s$ over a distance of $\Delta x$ on the water surface is

$$
c=\frac{\Delta s}{\Delta x}=1.1 \mathrm{~m}^{-1} \rightarrow R=\frac{1}{c}=0.91 \mathrm{~m} .
$$

Even for the very small error in height of $1 \mathrm{~mm}$, the maximum curvature radius is almost $1 \mathrm{~m}$. During the experiment, this curvature was exceeded even at the lower wind speeds. At $3 \mathrm{~m} \mathrm{~s}^{-1}$ wind speed, the dominant wave frequency in the Marseille facility is $f=4 \mathrm{~Hz}$, corresponding to a wavenumber of approximately $k=65 \mathrm{rad} \mathrm{m}^{-1}$ (not accounting for surface drift velocity). The dominant wave amplitude is $a=0.0016 \mathrm{~m}$. Assuming sinusoidal waves, this gives a curvature $c=a k^{2}=6.76 \mathrm{~m}^{-1}$, exceeding the determined minimum curvature for height errors less than $1 \mathrm{~mm}$. At higher wind speeds, capillary waves are formed which have much higher curvatures, so the error is certainly negligible. In any case, the total error of the height measurement is dominated by the error of assuming a constant water elevation in the interrogation window $(4 \times 4 \mathrm{~cm}$ in the laboratory, typically $20 \times 20 \mathrm{~cm}$ in the field). However, the goal is just to measure the waves around the spectral peak and to determine whether or not there is swell present in addition to locally generated wind waves, so this error is acceptable. It should be noted that the slope error described above (and in consequence the height error) will increase if the measurement footprint is increased. This error can be avoided if the light sources are placed at virtually the same location as the camera. One possibility to achieve this kind of coaxial lighting is using beam splitters in front of the cameras. For most applications, this will not be necessary as the error is dominated by the finite size of the interrogation window.

\section{References}

Banner M L, Jones I S F and J C Trinder 1989 Wavenumber spectra of short gravity waves J. Fluid Mech. 198 321-44
Benetazzo A 2006 Measurements of short water waves using stereo matched image sequences Coast. Eng. 53 1013-32

Benetazzo A, Fedele F, Gallego G, Shih P-C and Yezzi A 2012 Offshore stereo measurements of gravity waves Coast. Eng. 64 127-38

Bock E J and Hara T 1992 Optical measurements of ripples using a scanning-laser slope gauge: part II data analysis and interpretation from a laboratory wave tank Proc. SPIE 1749 272-82

Bréon F M and Henriot N 2006 Spaceborne observations of ocean glint reflectance and modeling of wave slope distributions J. Geophys. Res. 111005

Coantic M and Bonmarin P 1975 The air-sea interaction simulation facility at the institut de mecanique statistique de la turbulence Atmos. Technol. 7 72-9

Cote L, Davis J, Marks W, McGough R, Mehr E, Pierson W Jr, Ropek J, Stephenson G and Vetter R 1960 The directional spectrum of a wind generated sea as determined from data obtained by the stereo wave observation project Meteorol. Pap. 2 1-88

Cox C and Munk W 1954a Statistics of the sea surface derived from sun glitter J. Mar. Res. 13 198-227

Cox C and Munk W 1954b Measurements of the roughness of the sea surface from photographs of the sun's glitter J. Opt. Soc. Am. 44 838-50

Cox C and Zhang X 2011 Contours of slopes of a rippled water surface Opt. Express 19 18789-94

Cox C S 1958 Measurement of slopes of high-frequency wind waves J. Mar. Res. 16 199-225

Dobson E B 1970 Measurements of the fine-scale structure of the sea J. Geophys. Res. 75 2853-6

Edson J B et al 2013 On the exchange of momentum over the open ocean J. Phys. Oceanogr. 43 1589-610

Fedele F, Benetazzo A, Gallego G, Shih P-C, Yezzi A, Barbariol F and Ardhuin F 2013 Space-time measurements of oceanic sea states Ocean Model. 70 103-15

Frew N et al 2004 Air-sea gas transfer: its dependence on wind stress, small-scale roughness and surface films J. Geophys. Res. $109 \mathrm{C} 08 \mathrm{~S} 17$

Gallego G, Yezzi A, Fedele F and Benetazzo A 2011 A variational stereo method for the three-dimensional reconstruction of ocean waves IEEE Trans. Geosci. Remote Sens. 49 4445-57

Guizar-Sicairos M, Thurman S T and Fienup J R 2008 Efficient subpixel image registration algorithms Opt. Lett. 33 156-8

Hartley R and Zisserman A 2003 Multiple View Geometry in Computer Vision 2nd edn (Cambridge: Cambridge University Press) 
Helmholtz H 1867 Handbuch der Physiologischen Optik (Leipzig: Voss)

Holthuijsen L H 1983a Observations of the directional distribution of ocean-wave energy in fetch-limited conditions J. Phys. Oceanogr. 13 191-207

Holthuijsen L H 1983b Stereophotography of ocean waves Appl. Ocean Res. 5 204-9

Hughes B A, Grant R W and Chappell R W 1977 A fast response surface-wave slope meter and measured wind-wave moments Deep-Sea Res. 24 1211-23

Hwang P, Trizna D B and Wu J 1993 Spatial measurements of short wind waves using a scanning slope sensor Dyn. Atmos. Oceans $201-23$

Jähne B and Reimer K 1990 Two-dimensional wave number spectra of small-scale water surface waves J. Geophys. Res. 95 11531-646

Jähne B 1993 Spatio-Temporal Image Processing: Theory and Scientific Applications (Lecture Notes in Computer Science vol 751) (Berlin: Springer)

Jähne B 2005 Digital Image Processing 6th edn (Berlin: Springer)

Jähne B, Münnich K O, Büsinger R, Dutzi A, Huber W and Libner P 1987 On the parameters influencing air-water gas exchange J. Geophys. Res. 92 1937-50

Jähne B, Klinke J and Waas S 1994 Imaging of short ocean wind waves: a critical theoretical review J. Opt. Soc. Am. A 11 2197-209

Kiefhaber D 2010 Development of a reflective stereo slope gauge for the measurement of ocean surface wave slope statistics Diplomarbeit Institut für Umweltphysik, Fakultät für Physik und Astronomie, University Heidelberg

Kiefhaber D 2014 Optical measurement of short wind waves: from the laboratory to the field Dissertation Institut für Umweltphysik, Fakultät für Physik und Astronomie, University Heidelberg

Kiefhaber D, Reith S, Rocholz R and Jähne B 2014 High-speed imaging of short wind waves by shape from refraction J. Eur. Opt. Soc. Rap. Public 914015

Kinsman B 1965 Wind Waves: Their Generation and Propagation on the Ocean Surface (Englewood Cliffs, NJ: Prentice-Hall)

Kohlschütter E 1906 Die forschungsreise S.M.S. Planet II. Stereophotogrammetrische Aufnahmen Ann. Hydrogr. Maritimen Meterologie 34 220-7

Kosnik M V and Dulov V A 2011 Extraction of short wind wave spectra from stereo images of the sea surface Meas. Sci. Technol. 22015504

Kundu P K 2008 Fluid Mechanics 4th edn (New York: Academic)

Laas W 1905 Photographische Messung der Meereswellen Z. Ver. Dtsch. Ing. 49 S.1889 ff. S.1937 ff. und S.1976 ff

Laas W 1906 Messung der Meereswellen und ihre Bedeutung für den Schiffsbau Jahrbuch Schiffsbautechn. Ges. 7 S.391 ff

Lange P A, Jähne B, Tschiersch J and Ilmberger I 1982 Comparison between an amplitude-measuring wire and a slope-measuring laser water wave gauge Rev. Sci. Instrum. 53 651-5

Longuet-Higgins M S 1963 The effect of non-linearities on statistical distributions in the theory of sea waves J. Fluid Mech. 17 459-80
Mironov A S, Yurovskaya M V, Dulov V A, Hauser D and Guérin C A 2012 Statistical characterization of short wind waves from stereo images of the sea surface J. Geophys. Res. 117 C00J35

Schooley A H 1954 A simple optical method of measuring the statistical distribution of water surface slopes J. Opt. Soc. Am. 44 37-40

Schumacher A 1939 Stereophotogrammetrische Wellenaufnahmen, Ozeanographische Sonderuntersuchungen Wissenschaftliche Ergebnisse der Deutschen Atlantischen Expedition auf dem Forschungs- und Vermessungsschiff Meteor ed A Defant (Berlin: de Gruyter)

Schumacher A 1950 Stereophotogrammetrische Wellenaufnahmen mit schneller Bildfolge Dtsch. Hydrographische Z. 3 78-82

Schumacher A 1952 Results of exact wave measurements (by stereophotogrammetry) with special reference to more recent theoretical investigations Proc. NBS Semicentennial Symp. on Gravity Waves held at the NBS (18-20 June 1951) pp 69-78

Shaw J A and J H Churnside 1997 Scanning-laser glint measurements of sea-surface slope statistics Appl. Opt. $364202-13$

Shemdin O H, Tran H M and Wu S C 1988 Directional measurements of short ocean waves with stereophotography J. Geophys. Res. 93 13891-901

Stilwell D J 1969 Directional energy spectra of the sea from photographs J. Geophys. Res. 74 1974-86

Valenzuela G R 1978 Theories for the interaction of electromagnetic and ocean waves: a review Bound.-Layer Meteorol. 13 61-85

Veron F, Melville W K and Lenain L 2009 Measurements of ocean surface turbulence and wave-turbulence interactions $J$. Phys. Oceanogr. 39 2310-23

Waas S and Jähne B 1992 Combined slope-height measurements of short wind waves: first results from field and laboratory measurements Proc. SPIE 1749 295-306

Wu J 1971 Slope and curvature distribution of wind-disturbed water surface J. Opt. Soc. Am. 61 852-8

Zappa C, Banner M, Schultz H, Corrada-Emmanuel A, Wolff L and Yalcin J 2008 Retrieval of short ocean wave slope using polarimetric imaging Meas. Sci. Technol. 19055503

Zappa C J, Asher W E, Jessup A T, Klinke J and Long S R 2004 Microbreaking and the enhancement of air-water transfer velocity J. Geophys. Res. 109 C08S16

Zappa C J, Banner M L, Schultz H, Gemmrich J, Morison R P, LeBel D and Dickey T 2012 An overview of sea state conditions and air-sea fluxes during $\mathrm{RaDyO} J$. Geophys. Res. $117 \mathrm{C} 00 \mathrm{H} 19$

Zhang X, Dabiri D and Gharib M 1996 Optical mapping of fluid density interfaces: concepts and implementations Rev. Sci. Instrum. 67 1858-68

Zickler T, Belhumeur P and Kriegman D 2002 Helmholtz stereopsis: exploiting reciprocity for surface reconstruction Int. J. Comput. Vis. 49 215-27 